\title{
Correlative In-Situ Analysis on the Nanoscale by combination of AFM and SEM
}

M. Winhold ${ }^{1}$, M. Leitner ${ }^{1}$, P. Frank ${ }^{1}$, N. Hosseini ${ }^{2}$, J. Sattelkov ${ }^{3}$, G.E. Fantner ${ }^{2}$, H. Plank ${ }^{3}$ and C.H. Schwalb ${ }^{1}$

1. GETec Microscopy GmbH, Vienna, Austria.

2. Laboratory for Bio- and Nano-Instrumentation, Institute for Bioengineering, EPFL, Switzerland.

3. Institute for Electron Microscopy and Nanoanalysis, Graz University of Technology, Austria.

The combination of different analytical methods into one instrument is of great importance for the simultaneous acquisition of complementary information. Especially the in-situ combination of scanning electron microscopy (SEM) and atomic force microscopy (AFM) enables completely new insights in the micro and nano world. In this work, we present a novel AFM - the AFSEM - that can be easily integrated into the high-vacuum environment of almost any scanning electron microscope (SEM) or other host system (e.g. dual beam microscopes). It allows direct in-situ combination of these complementary techniques due to the simultaneous operation of SEM and AFM inside the vacuum chamber.[1,2] Therefore, SEM imaging, chemical information by EDX, real 3D topography, phase information, mechanical, electrical, and magnetic properties by AFM can be combined in an easy and interactive way. Furthermore, due to the open design it can be combined with additional add-ons, e.g., tensile stages, nano-indentors[2] or nano-manipulators (Figure 1a).

We will present a variety of case studies to highlight the advantages of correlative in-situ analysis for different materials. We show results for in-situ electrical characterization of nanostructures by combining SEM and conductive AFM measurements for dose-dependent conductivity measurements of nanogranular $\operatorname{Pt}(\mathrm{C})$ structures after focused electron beam induced processing (Figure 1b). In addition, we will demonstrate the analysis of bone tissue and show how the SEM allows to quickly identify the area of interest, in this case the lacunae on the surface of the bone structure (Figure 2a). The AFM then provides the real 3D topography inside the lacunae (Figure $2 \mathrm{~b}$ ) and enables the detailed analysis of the collagen fibers within the lacunae area (Figure $2 \mathrm{c}+\mathrm{d}$ ). Finally, we show results for monolayer growth characterization of 2D materials, failure mechanic analysis by in-situ tensile stress measurements of metal wires or polymer structures.

Based on the broad variety of applications regarding the characterization of different materials and devices we anticipate the AFSEM to be one of the driving characterization tools for correlative $\mathrm{SEM} / \mathrm{AFM}$ analysis in the future.

\section{References:}

[1] D. Yablon, P. Werten, M. Winhold and C.H. Schwalb, Microscopy and Analysis 31(2) 14-18 (2017) [2] J. Kreith, T. Strunz, E.J. Fantner, G.E. Fantner and M.J. Cordill, Rev. Sci. Instr. 88053704 (2017) 
(a)

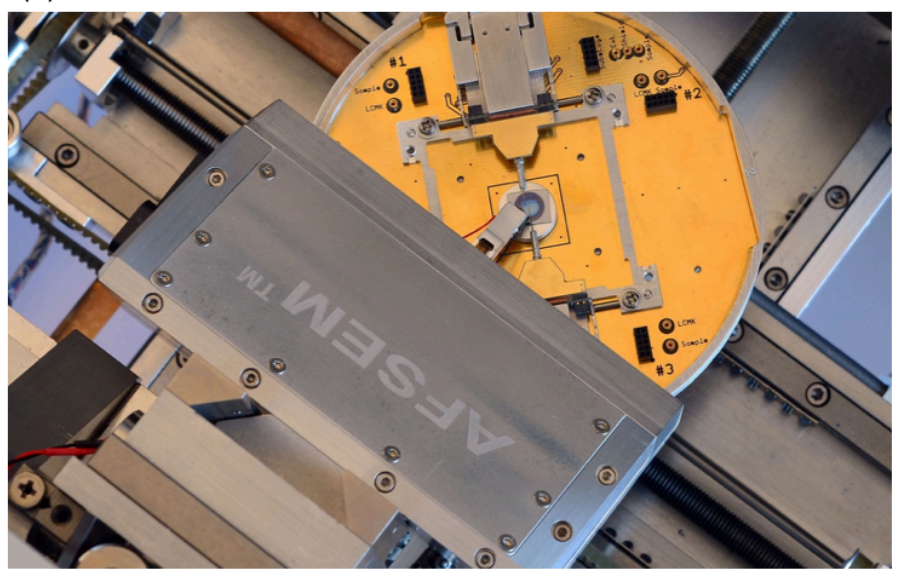

(b)
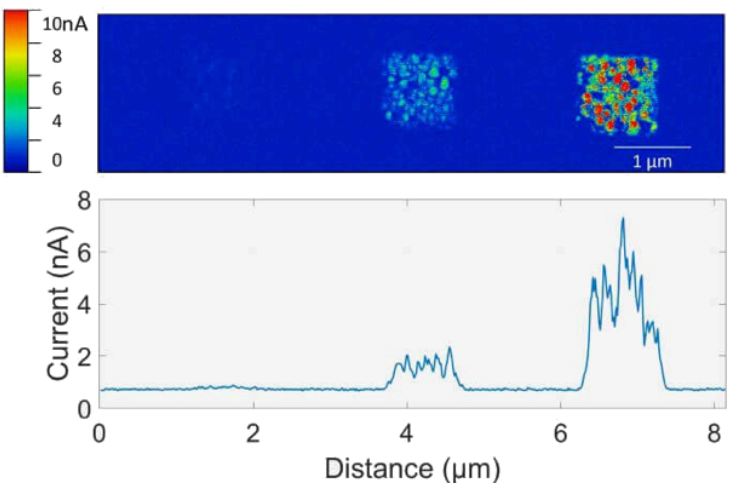

Figure 1. (a) AFSEM integration inside SEM in combination with a nanomanipulator setup (b) In-situ AFM topography of nanogranular $\operatorname{Pt}(\mathrm{C})$ structure prepared by focused electron beam induced deposition (c) In-situ conductive AFM measurement of nanogranular $\mathrm{Pt}(\mathrm{C})$ structure.
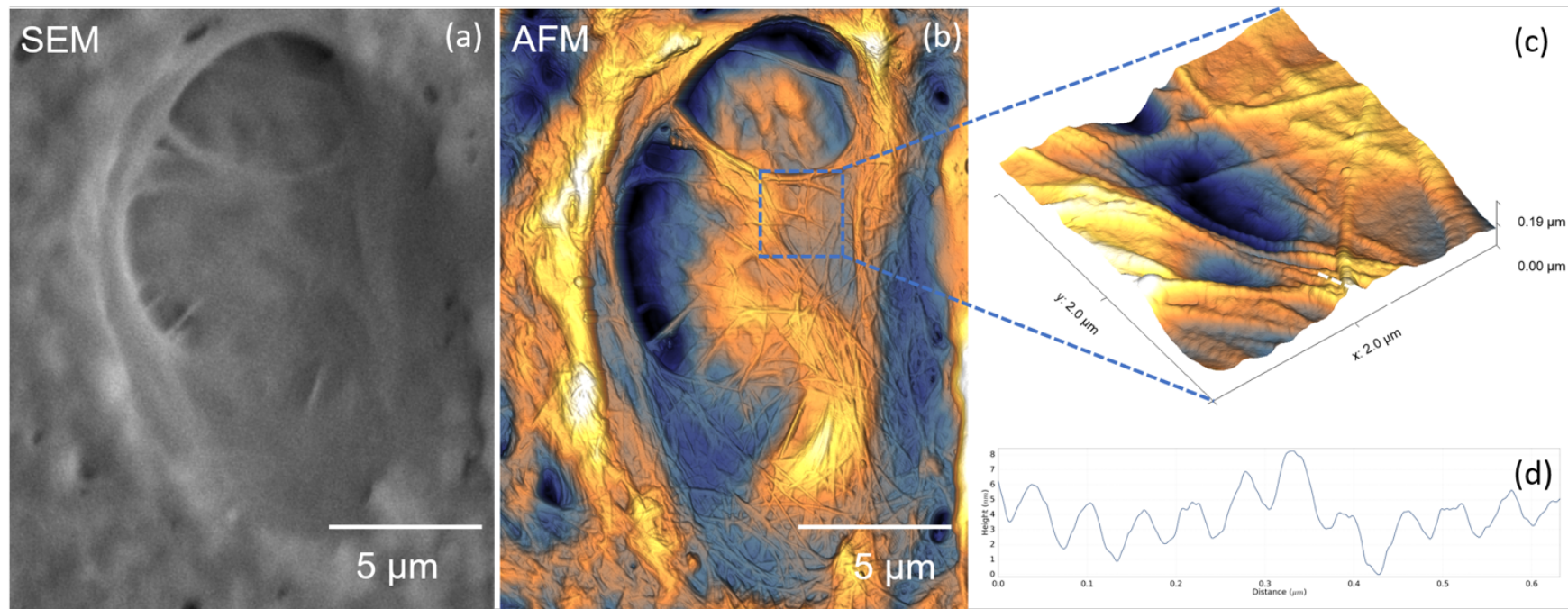

(c)

Figure 2. (a) SEM image of lacunae structure on a bone tissue surface. (b) Correlative in-situ AFM image of lacunae structure. (c) High-resolution AFM image of collagen fibers inside the lacunae. (d) Cross section of collagen fiber surface structure. 